\title{
Digital Image Correlation Techniques Applied to Large Scale Rocket Engine Testing
}

\author{
Paul R. Gradl ${ }^{1}$ \\ NASA Marshall Space Flight Center, Huntsville, AL 35812
}

Rocket engine hot-fire ground testing is necessary to understand component performance, reliability and engine system interactions during development. The $\mathrm{J}-2 \mathrm{X}$ upper stage engine completed a series of developmental hot-fire tests that derived performance of the engine and components, validated analytical models and provided the necessary data to identify where design changes, process improvements and technology development were needed. The J-2X development engines were heavily instrumented to provide the data necessary to support these activities which enabled the team to investigate any anomalies experienced during the test program. This paper describes the development of an optical digital image correlation technique to augment the data provided by traditional strain gauges which are prone to debonding at elevated temperatures and limited to localized measurements. The feasibility of this optical measurement system was demonstrated during full scale hot-fire testing of $\mathbf{J}-2 \mathrm{X}$, during which a digital image correlation system, incorporating a pair of high speed cameras to measure three-dimensional, real-time displacements and strains was installed and operated under the extreme environments present on the test stand. The camera and facility setup, pre-test calibrations, data collection, hot-fire test data collection and post-test analysis and results are presented in this paper.

\section{Nomenclature}

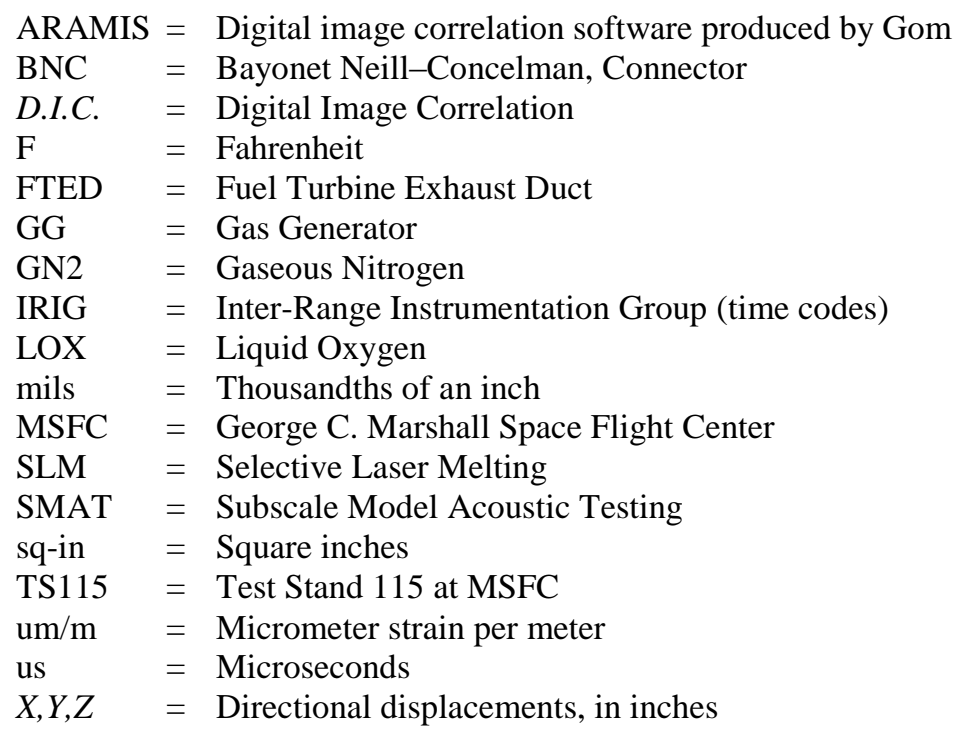

${ }^{1}$ Combustion Devices Design and Manufacturing Engineer, NASA Marshall Space Flight Center, Huntsville, AL 35812 


\section{Introduction}

$\mathrm{H}$ OT-FIRE engine testing requires a variety of instrumentation to determine engine performance, monitor health, and provide design data for further improvements and upgrades. This instrumentation includes pressure transducers, thermocouples, accelerometers, strain gages, and can include other specialty instrumentation. Although this instrumentation has improved with modern sensors, the basics of the instrumentation has remained relatively unchanged. One commonality between all the traditional instrumentation installed is that discrete ports or single point data probes are used to collect data, which provides a localized indication of conditions. Instrumentation locations must be selected based on priority locations to accurately map component and overall engine performance. Modern techniques have been developed and advanced using three-dimensional (3D) sensor techniques that can collect a vast quantity of full surface data in the time domain. One of these techniques that has been explored for engine testing is Digital Image Correlation (D.I.C.).

Development of digital image correlation is being pursued for data collection of elevated temperature components such as Carbon-Carbon and metallic nozzle extensions, fluid transfer ducts and other components. This allows for hot-fire test data to be collected and finite element modeling techniques anchored using high fidelity test data. These measurements and modeling techniques have traditionally been difficult to obtain on Carbon-Carbon extensions and other high temperature components. This enables models to be validated and a reduction in uncertainties to potentially minimize testing and reduce associated costs. Digital Image Correlation techniques are also being explored for cryogenic applications which expands the use of this technique beyond room and elevated temperature applications ${ }^{1}$.

Digital image correlation is a three dimensional imaging technique that measures deformations and surface strains in the time-domain. This technique allows for full surface engineering data to be collected of objects under static and dynamic loading by correlating sub-pixel patterns to determine displacements of the object being measured ${ }^{2}$. Standard frame rate or high speed digital imaging sensors can be used for paired image data collection. The setup and calibration techniques for digital image correlation are similar to those of structured light scanning using similar pre-determined optimum camera angles and similar calibration artifacts. However, the digital image correlation uses a stochastic high contrasting speckle pattern that is applied directly to the surface of the object being measured. The direct application of this stochastic speckle pattern allows for unique sub-pixel correlations from one image to the other within the pair and subsequent displacements and surface strains (change in length per the original length) to be calculated ${ }^{3}$.

Digital image correlation collects a pair of images at each instance of time, which is also known as a stage. Data can only be collected and analyzed that is common to each camera's overlapping field of view. Using the calibration data and similar triangulation formulas to structured light, a three dimensional surface, or mesh, can be determined for each stage of time. For determining surface deformation, a mesh surface can be compared back to an initial baseline or different stage within the sequence, providing a three-dimensional color plot and delta of the surface normals. This visual data allows for a more intuitive evaluation of engineering data and hardware performance.

There were several motivations to advance digital image correlation for rocket engine testing. This technique is a non-contact optical technique and can be deployed and calibrated rapidly. It provides non-intrusive full-field measurements to evaluate surface strains, displacements, and accelerations. This provides a direct replacement for traditional instruments that could only collect discrete data points. Digital image correlation also provided a local and global view of the hardware and easily interpreted results to understand trends, hardware loading, and provide for performance and life predictions.

One of the main purposes for pursuing digital image correlation for engine testing was the installation challenges of existing instrumentation. Several components during engine testing experience elevated temperatures that limit the use of certain bonding techniques for strain gages and accelerometers. The preferred strain gages (non-weldable) are bonded using adhesives that degrade above temperatures a few hundred degrees Fahrenheit and, in most environments, will debond. This limits the usage of these gages to the start transient and data is often unreliable due to early detachment. Additional post-test processing and data filtering for strain gages is often necessary to eliminate unnecessary noise and properly interpret the data. Filtering is not completely eliminated with digital image correlation, but is greatly simplified within the D.I.C. software. Accelerometers can be threaded or bonded, but have 
limitations in the directional orientation that data is collected. Digital image correlation can provide $\mathrm{X}, \mathrm{Y}, \mathrm{Z}$ (tri-axial) data in addition to rotational degrees (six degrees of freedom), if necessary, when properly set up.

The digital image correlation system provides an ease of deployment to collect line-of-sight strain and displacement data. With each traditional strain gage or accelerometer installed, the physical cabling must be run and tied back to a data system. Discrete gages have to be physically bonded to the part and properly zeroed while online and offline noise filtering is completed to insure unbiased or "clean" data. The setup for D.I.C. includes installation and calibration of the cameras and speckling of the components to be imaged. The typical setup, installation, and calibration of the cameras can be completed in a matter of hours and, in most cases, completed the morning of test. The stochastic pattern speckling can also be completed in a matter of hours. The ARAMIS and PONTOS ${ }^{4,5}$ software packages were selected to be used for the digital image correlation and photogrammetry imaging techniques for compatibility with existing datasets residing in corollary Gom products.

The D.I.C. technology does have certain limitations. The most significant limitation is that both cameras must have direct line of sight to obtain paired data for processing. Since the technology works on triangulation principles, the cameras are calibrated as a pair to obtain 3D surface data. This requires that the cameras have field of view that overlaps in the region of interest being imaged. This limits data to be collected using D.I.C. where data locations are fully obstructed or obscured by other components, small lines or other instrumentation lines. Debris blocking one or both cameras during the time period of interest may also limit temporary data collection. Another limitation of D.I.C. is highly elevated temperatures (above $1600^{\circ} \mathrm{F}$ ), although techniques are being explored to resolve these issues and research has shown solutions to this issue ${ }^{6}$. Since D.I.C. is an optical measurement system, the surface must not only be visible, but the contrast with the stochastic pattern must be apparent. Otherwise, when components reach elevated temperatures, the cameras can be oversaturated and the contrast between the black and white pattern is lost and data not obtainable. Techniques are being investigated, though, to make use of filtering techniques to and suppressing black body radiation for elevated temperatures ${ }^{7,8}$. Additional research is being conducted to view surfaces outside of the visible spectrum that build upon the previous blue light method and may be pursued for such applications, as proposed by Burke and Lambros ${ }^{9}$.

\section{Initial Development Testing}

A significant challenge presented for Digital Image Correlation techniques is the highly dynamic and harsh environments of rocket engine testing. Prior to moving into full scale testing, a series of subscale tests were completed to develop techniques, stabilizing systems, stochastic paints and patterns. and process flow. Since testing was targeting elevated temperature components, a parallel development effort was initiated to lab test and down select the appropriate paints. Initial digital image correlation testing was completed in the laboratory, which simulated setups such as cameras and mock components to optimize the process flow prior to moving to the test stands. Early hot-fire data collection was completed during F-1 Gas Generator testing at Marshall Space Flight Center Test Stand 116, as well as Space Launch System (SLS) Subscale Model Acoustic Testing (SMAT) combustion chamber hot-fire checkouts at TS115. These smaller test beds provided the opportunity to checkout camera setup and stability, lens stability, calibration techniques, painting techniques and general process flow.

A methodical development process was applied to evaluate the high temperature paints that were to be used on the components being measured. A variety of off the shelf paints were evaluated for adherence to the base materials being evaluated, reliability at exposed temperatures, visible photogrammetric properties and material compatibility. Application techniques for the paints were developed, focusing on aerosol spray cans, hobby-grade pneumatic spray guns and a manual template/maskant-based technique for larger stochastic patterns in low volumes. A variety of stochastic patterns for small applications (less than 144 sq-in) can be applied using basic techniques with the aerosol cans and spray guns. For the larger applications, basic plastic templates were used. For more complex surfaces, however, adhesive vinyl templates were required.

The paint patterns were applied to a series of A-286 stainless steel and Inconel 625 substrate panels to evaluate adherence and temperature exposure. The panel substrate specimens were exposed in an air furnace at a series of increasing temperature runs that were selected based on the use applications for interim and peak temperature. Rapid heat testing was not evaluated during the initial exposure testing. The panels were evaluated following each exposure for adherence to the substrate and the ability to maintain a contrasting pattern. Photogrammetry was completed following each of the exposures to determine if the paint (1) was suitable for use during elevated digital 
image correlation and dynamic photogrammetry, and (2) provided stable contrast. Material compatibility testing was also completed on the down- selected paints after they completed the furnace exposure testing. Materials were tested for sulfur and chloride content that could adversely affect the substrate material.

Paints down-selected for higher temperature applications, and subsequently evaluated for durability, photogrammetric capabilities and peak temperature exposure, are shown in Table 1. The temperature limits were based solely on exposure testing and did not consider any bandpass filtering techniques that could increase theoretical use limits ${ }^{10}$.

Table 1. High Temperature Paints Tested for Hot-fire Testing Applications.

\begin{tabular}{cc}
\hline \hline Paint Name & Temperature Limit, $^{\circ} \mathbf{F}$ \\
\hline Hi-Temp Coatings, 1000 VS-902 Cloudy Gray & 1000 \\
Hi-Temp Coatings, 1000 VS-803 Crème & 1000 \\
Rustoluem Flat Black 1976 & 1000 \\
Zynolyte Hi Temp, Z642 Machinery Gray & 1200 \\
Zynolyte Hi Temp, Z645 White & 1200 \\
VHT Flameproof, SP102 Black & 1600 \\
VHT Flameproof, SP101 White & 1600 \\
Aluminum Oxide Powder, Binder Suspended & 1700 \\
\hline \hline
\end{tabular}

A series of feasibility tests were conducted at MSFC TS115 to evaluate camera-facility integration, camera and lens stability, paint stability and general process flow. The components being evaluated were radiatively-cooled, truncated nozzles fabricated using Inconel 625 additive manufacturing techniques, specifically selective laser melting (SLM) shown in Figure 1. The nozzles were thinned to thicknesses of 0.020-0.040" (20-40 mils) to allow for additional deflections to be observed during the test. A pair of Vision Research Phantom v7 high speed cameras were used to complete the test series. A pair of $85 \mathrm{~mm}$ fixed focus lenses were mounted to the cameras. The camera system was then mounted to lockable brackets and extruded aluminum bar at approximately 5 feet from the nozzle test article. The trigger signal and IRIG timing signals were applied by the test facility via BNC cables to the high speed cameras. All setup and calibrations were completed the morning of testing.
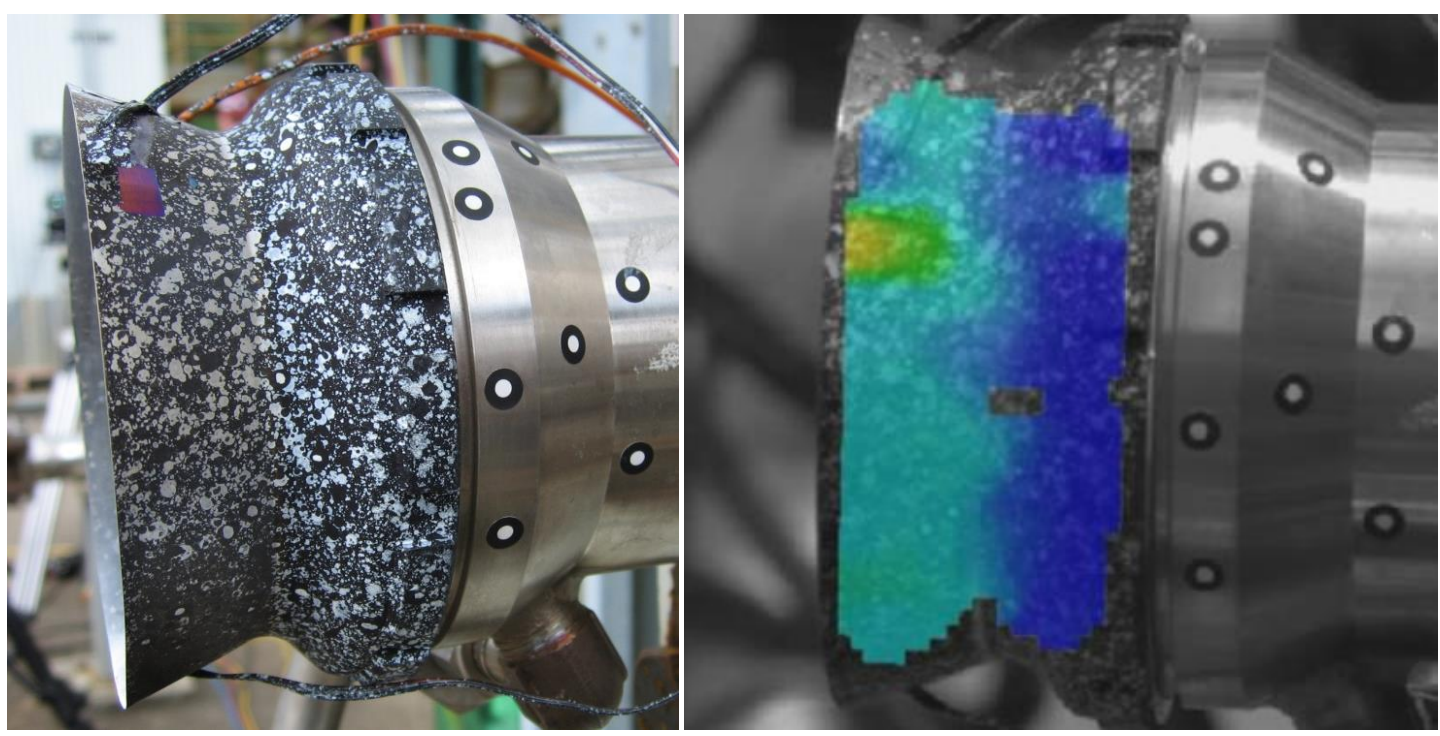

Figure 1. Subscale feasibility nozzle testing at MSFC Test Stand 115, Left Image is a static picture of the nozzle post-test; Right image is a stage during steady state hot-fire operation showing radial displacement relative to pre-hot-fire condition. 
Original test planning included a series of protective shields to be installed in front of the cameras. However, it was discovered during lab testing that the polycarbonate was causing significant distortions during calibrations and unacceptable errors. The high quality, bubble-free polycarbonate originally evaluated had a thickness of 0.5 " which caused significant errors during calibrations. A thickness of 0.25 " was evaluated and allowed a successful calibration, but still increased error. It was discovered a thickness of 0.125 " was acceptable and minimized calibration and data collection errors, but caused additional concerns of flutter from vibrations or shocks during the hot-fire tests. It was decided to forego the protective shields during the initial testing to minimize sources of error.

Initial data collection was not successful due to an adherence failure of the paint. The paint was originally applied using a manual speckling technique and was too thick in most locations causing it to spall from the rapid heating of the nozzle. It was concluded from the initial testing that the paint needed to be maintained at a thickness of less than 0.005 " (5 mils) to allow for proper adherence to the component. This was also later confirmed with additional furnace exposure testing where cracking was observed with thicker applications of the paint. The paint was reapplied to the subsequent nozzles using the spatter technique from the aerosol cans. Due to a finer speckle pattern, the lenses were changed to $105 \mathrm{~mm}$ to provide for a smaller field of view to obtain the optimal pixel distribution later used in the facet construction within the software.

The subscale hot-fire testing demonstrated the ability to measure elevated temperature components using digital image correlation and developed a generic process flow for the camera setup and calibration techniques ${ }^{11}$. The system was unable to collect data above approximately $1700^{\circ} \mathrm{F}$ due to overexposure from the glow of the radiatively cooled nozzles as they increased in temperature, rejecting heat. The measured radial deflections, driven by the radial thermal growth, tracked and closely matched the predictions, confirming the system's ability to measure at elevated temperatures. The raw data shown in Figure 2 from D.I.C measurements was unfiltered. A strain gage was attached to the nozzle to allow for comparison data, but detached at approximately $220^{\circ} \mathrm{F}$ early in the test, so strain comparison data was not available. This strain gage failure further supported the development need for digital image correlation for these types of elevated temperature applications.

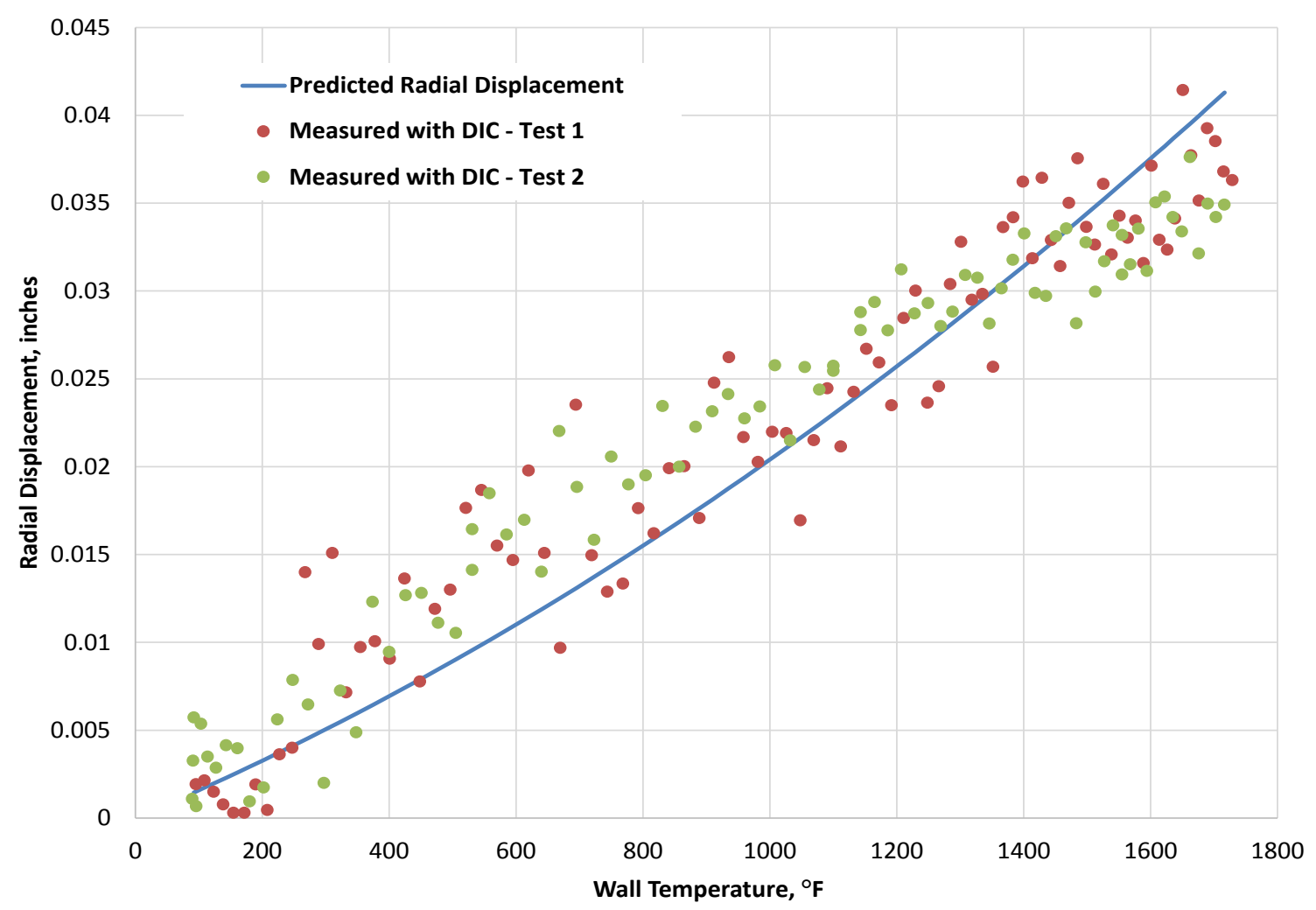

Figure 2. Predicted and Actual (Unfiltered Raw) Radial displacement as measured using digital image correlation on subscale nozzle. 
The radial displacements show a slight under-prediction at mid-range temperatures. There were additional dynamics of the actual nozzle and thrust chamber not apparent in this dataset that affect some of the localized and global movements. When a 6-period moving average is applied to the dataset, it tracks closer to the predictions and less than 0.003" (3 mils) of deviation is observed between predicted and actual. This is still large compared to the overall movement, but provides an indication of the precision of the measurement at elevated temperatures.

\section{Full-scale Development Feasibility Testing}

\section{A. Hardware Setup}

To verify the capabilities of the D.I.C. technique during full-scale engine operation, the J-2X engine program funded its use during hot-fire testing over a period from July 31, 2013 through September 5, 2013, on the J-2X development engine. The development of digital image correlation techniques was being funded by and conducted under the J-2X engine program ${ }^{12}$. Engine 10002 at the Stennis Space Center (SSC) A1 test stand was targeted to prove the feasibility of this technique in a full-scale environment. The gimbal testing series was selected for D.I.C. evaluation because it provided additional challenges with radiant heating from the varying angles during gimballing and vibrations in the test stand. This provided the opportunity to develop all the necessary enclosures, camera techniques and support systems in a complex environment. The primary focus of the testing was to develop the technique and prove feasibility of full scale testing, so two elevated temperature ducts were targeted, the Gas Generator Exhaust Duct and the Fuel Turbine Exhaust Duct (FTED) shown in Figure 3. These had external surface temperatures beyond $500^{\circ} \mathrm{F}$. The gimbal testing provided the opportunity to obtain global displacement measurements of the engine, as well as localized displacements and engineering strains.

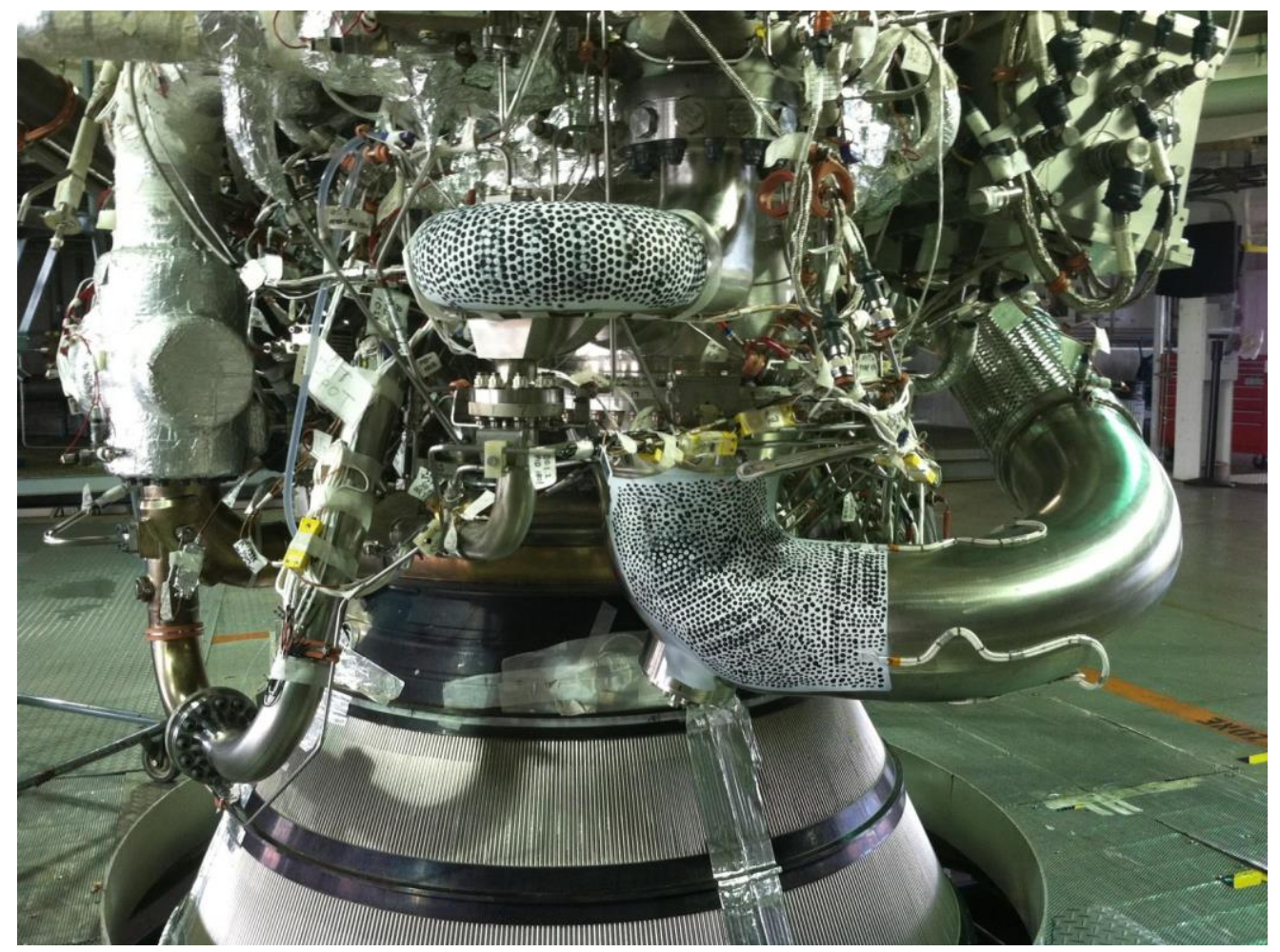

Figure 3. J-2X Engine 10002 with the speckled Gas Generator Duct and Fuel Turbine Exhaust Duct.

Setup for testing included a rigid steel support bar that was welded into the thrust take-out structure at 15 feet from the centerline of the engine, or approximately 12 feet from the exhaust ducts. The support structure was welded at a predetermined angle so the cameras could be aligned and the ducts placed central to the field of view. The angle of 17.5 degrees was optimized to meet the field of view required with full gimbal motion and also the proper angle for high accuracy D.I.C. A piece of angle iron provided the base for the camera setup and was welded to two steel pipes at either end and subsequently welded into the facility. An elastomer strip was then applied to the angle iron to 
help damp some of the loads. The extruded aluminum bar was bolted to this assembly through to the angle iron. Extruded aluminum bar was used to allow for the rotational brackets to be easily translated and locked into position, holding the cameras rigid. The original elastomer had a shore hardness of 40 , but due to loads causing a low frequency rock (tilt) of the paired cameras, a higher shore hardness material was used.

A pair of aluminum enclosures were fabricated that allowed for a positive GN2 purge of the cameras during testing shown in Figure 4. The back side of the enclosures had the associated BNC, Ethernet and power thruconnectors on the box to minimize leakage and maintain the positive purge. A high quality fused quartz window, 0.375 " in thickness, was captured between two O-rings and aluminum plates at the front of the enclosure. There were no issues with the quartz during any of the camera calibrations or testing. Previous enclosures that used Polycarbonate experienced higher errors in calibrations. The enclosures were bolted directly to rotational brackets to allow for the camera pan angles to be properly adjusted. Rotational brackets were locked in position using a series of hex head bolts. Enclosures were fabricated such that they were modular and access to the cameras was easy to allow for manual fine-focus and aperture adjustments to be made after camera pan positions were locked down. Once all adjustments were made to the cameras, the enclosure was bolted shut and the entire enclosure and paired bar assembly was wrapped in a thermal blanket and sealed with a high temperature tape. Temperature strips were applied to several locations on the camera housing and enclosure assembly to monitor temperatures post-test.
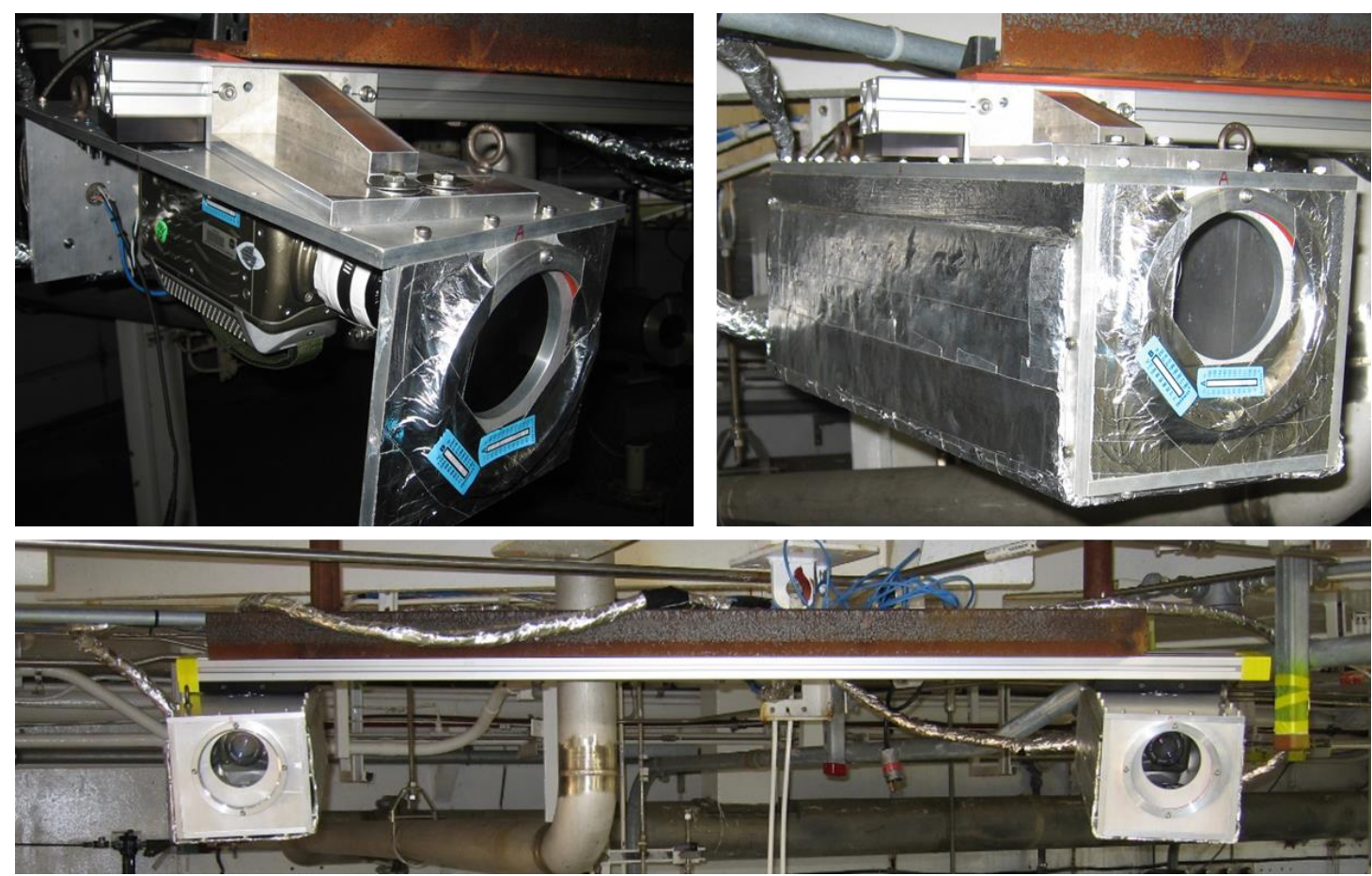

Figure 4. High Speed camera aluminum enclosures and setup hard mounted in the A1 test stand.

The paired Vision Research Phantom v7 cameras and PCC software were also used for this test series on the A1 test stand ${ }^{13}$. The enclosures were fabricated to fit this model as well as Phantom v411, which were in procurement at the time of testing. The v411 cameras were selected for the ability to use the high data transfer rate hard drives (Cinemag) that could record up to 760 frames/second for the entire duration of the 8.5 minute test. The cameras were synced in a master/slave mode and also tied in with the facility IRIG. A pair of 50mm fixed focus Nikkor lenses were used in the initial testing and later changed to 50mm Schneider Optics Xenon Emerald industrial lenses. This will be discussed later in the paper. Due to the complex environment of testing, a series of changes were made between each test to ensure proper data quality. The camera settings, lens configurations and other changes are noted in Table 2 below. 
Table 2. Camera Configuration for Engine 10002 Testing on A1.

\begin{tabular}{ccccccc}
\hline \hline Test & Date & $\begin{array}{c}\text { Test Duration } \\
\text { (sec) }\end{array}$ & \multicolumn{1}{c}{ Lens } & $\begin{array}{c}\text { Framerate } \\
\text { (frames/sec) }\end{array}$ & $\begin{array}{c}\text { Aperature } \\
\text { (f-stop value) }\end{array}$ & $\begin{array}{c}\text { Exposure } \\
\text { (us) }\end{array}$ \\
\hline A1J026 & $7 / 31 / 2013$ & 550 & $50 \mathrm{~mm}$ Nikkor & 60 & $\mathrm{f}-4$ & 800 \\
A1J027 & $8 / 15 / 2013$ & 885 & $50 \mathrm{~mm}$ Nikkor & 60 & $\mathrm{f}-4$ & 800 \\
A11028 & $8 / 23 / 2013$ & 550 & $50 \mathrm{~mm}$ Schneider & 60 & $\mathrm{f}-11$ & 40 \\
A11029 & $9 / 5 / 2013$ & 330 & $50 \mathrm{~mm}$ Schneider & 60 & $\mathrm{f}-11$ & 45 \\
\hline \hline
\end{tabular}

The first test, A1J026, used the Nikkor 50mm fixed focus lenses. The aperture was set to $\mathrm{f}-4$, which provided the appropriate depth of focus necessary. There were several issues discovered during and post-test that led to changes. The issue discovered during data collection and post-test was that the lenses had shaken loose due to high frequency vibrations on the stand. This occurred just after the start transient into main stage operation. Minimal data was available during the early start transient for processing. Data was also lost about 250 seconds into the test due to cables being burned up on the West engine deck from the excessive plume radiation due to the engine gimbaling. The initial fix for the lens vibrations between tests was to use a thin foam pad and standard hose clamp to lock the focus in position. However, the data still had issues with processing due to image blur, which was further evaluated during the second test. A high temperature fabric insulation and aluminum tape was also used on all data cables on the engine deck.

The clamped lenses were used for the second test. The lenses did not experience any defocusing issues during the test but image blur was still present in the data. This image blur was due to the exposure time being prolonged. A second issue discovered was the cameras experiencing a low frequency "rock" along the tilt axis (forward to back). Some of this tilt was in phase for the paired cameras, but there were some images out of phase, which would have caused issues during data processing. A series of changes were made between test A1J027 and A1J028, including additional changes to the lenses, exposure settings for the cameras, stiffening brackets and damping material between the welded steel support bar and the extruded aluminum bar.

A new pair of 50mm fixed focus lenses were installed for test A1J028. These were Schneider Optics machine vision Xenon Emerald lenses (f-stop 2.2, Part number 21-1062672). These lenses were custom fabricated with NDVibratite adhesive used during the assembly process to lock all internal optical components. In addition to this rigidity internally, the iris and focus rings were lockable with set screws. This provided several features for long term testing and eliminated any issues with the hose clamps. A major change observed with the Schneider lenses was the sensitivity and ability to set the aperture to 111 with the same lighting settings as the original Nikkor lenses. The exposure settings for the camera were a simple software change to a minimum exposure allowed with the available lighting on the test stand.

Two of the critical changes for test A1J028 were the forward and aft brackets and the change of the damping material. The rotational brackets were stiffened at the forward and aft end of each camera using angle brackets and bolted to the extruded aluminum bar and camera housings. These were installed following all alignments and prior to final focusing. The brackets stiffened the entire structure of the enclosures, brackets and extruded aluminum bar. A second change was made to the durometer of the damping material between the steel support angle iron bar and the extruded aluminum bar. A higher temperature, lower durometer material was used to minimize the low frequency rock and decouple the cameras from the facility. This allowed for some additional movement of the cameras, but would allow the camera movement to remain in phase. An image of the cameras during a hotfire test in shown in Figure 5. 


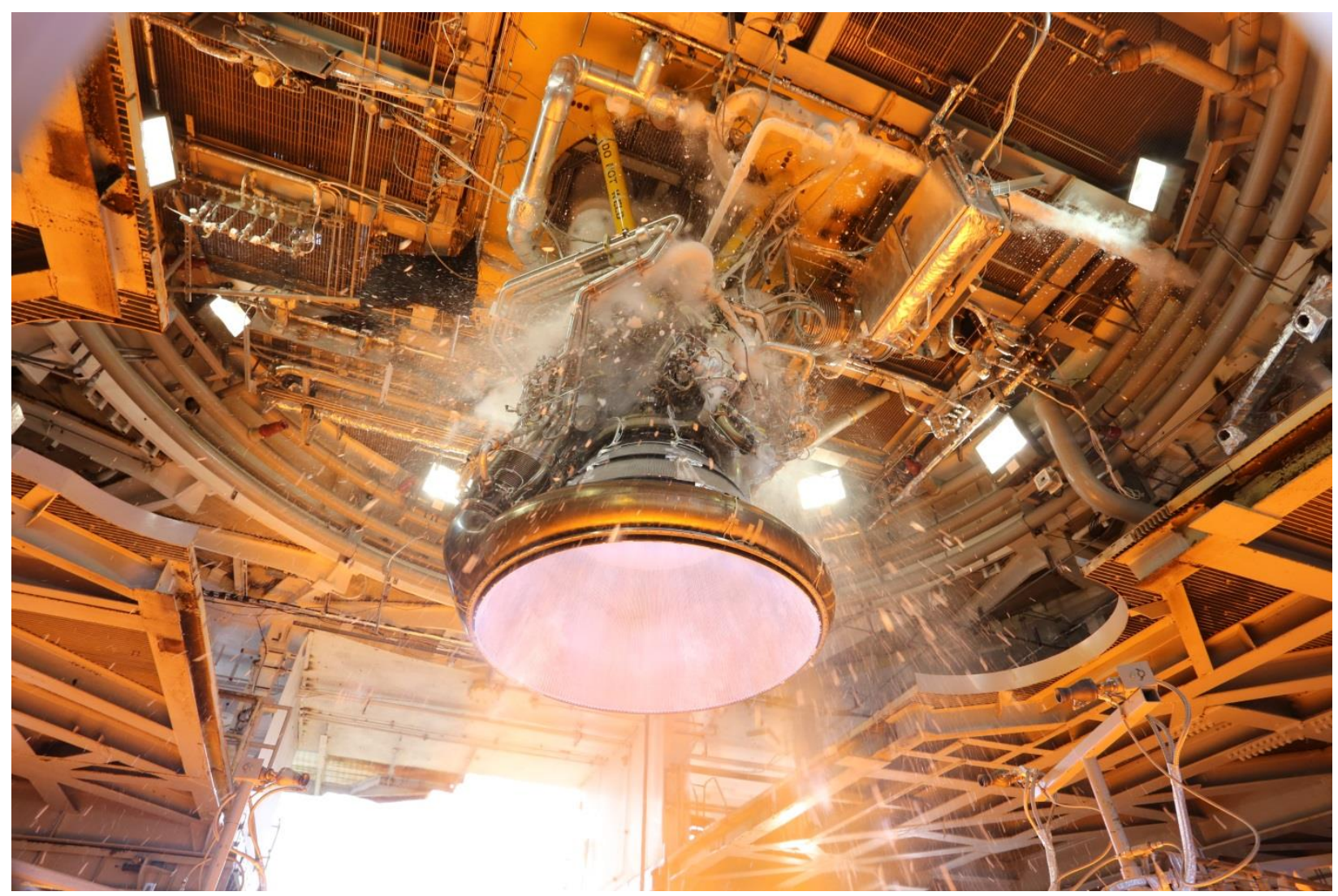

Figure 5. J-2X Engine 10002 during Hot-fire testing on A1. The Digital image Correlation high speed cameras are seen in the right-hand side of the picture.

Prior to the A1J028 test, it was also observed that the left camera had more movement than the right camera. Review of the hardware setup identified that the left camera was cantilevered out farther from the vertical to which the angle iron was welded, attaching it to the facility. The left camera was moved in slightly toward the centerline to maintain symmetry. The changes made prior to A1J028 provided a rigid setup and acceptable data for further digital image correlation processing.

\section{B. Data Collection and Processing}

Preparation for hot-fire testing included installation of the cameras the evening prior to test to minimize test day operations. This included all alignments, final focus, aperture settings and sealing of the camera enclosures with the GN2 purge. Preliminary calibrations were also completed and pre-test data collected to verify calibrations. Due to hazardous conditions present on the test stand during test day preparations, final calibrations were completed the morning of, approximately 8 hours prior to engine start. The cameras were networked so they could be controlled remotely during calibrations and also from the remote blockhouse during testing. A 1-meter calibration cross was used for calibrations which maximized the field of view. Calibrations were completed using a calibrated image series in the ARAMIS 6.3 software with 15 images. Calibrations were consistently less than 0.06 pixel deviation and $<0.0007^{\prime}$ " scale bar deviation for all tests. A camera angle of $17.5^{\circ}$ was calculated and measurement volume of approximately $54 \times 42 \times 42$ inches was within the calibrated field of view.

Test operations included a series of checkouts on the engine and test stand, loading of propellants, chilling and thermal conditioning of turbomachinery and valves, as well as installation of igniters and other preparations. Digital image correlation data was collected manually using a soft trigger at approximately every 30 minutes in addition to other significant events during the test countdown shown in Figure 6. This data validated that calibrations were maintained, but also provided tracking of the engine as it shifted when propellants were loaded ("dropped") and as the ducts and pumps were chilled. The LOX pump was positioned approximately 150 degrees opposite the GG exhaust duct and FTED being measured. The shift of the engine was apparent when LOX was dropped and the pump started chill operations. Data captured later in the pre-test sequence provided displacements as the ducts and 
the pumps chilled. Additional data observed the movements during activation of the gimbal actuators during a bump check, followed by damping of the engine.

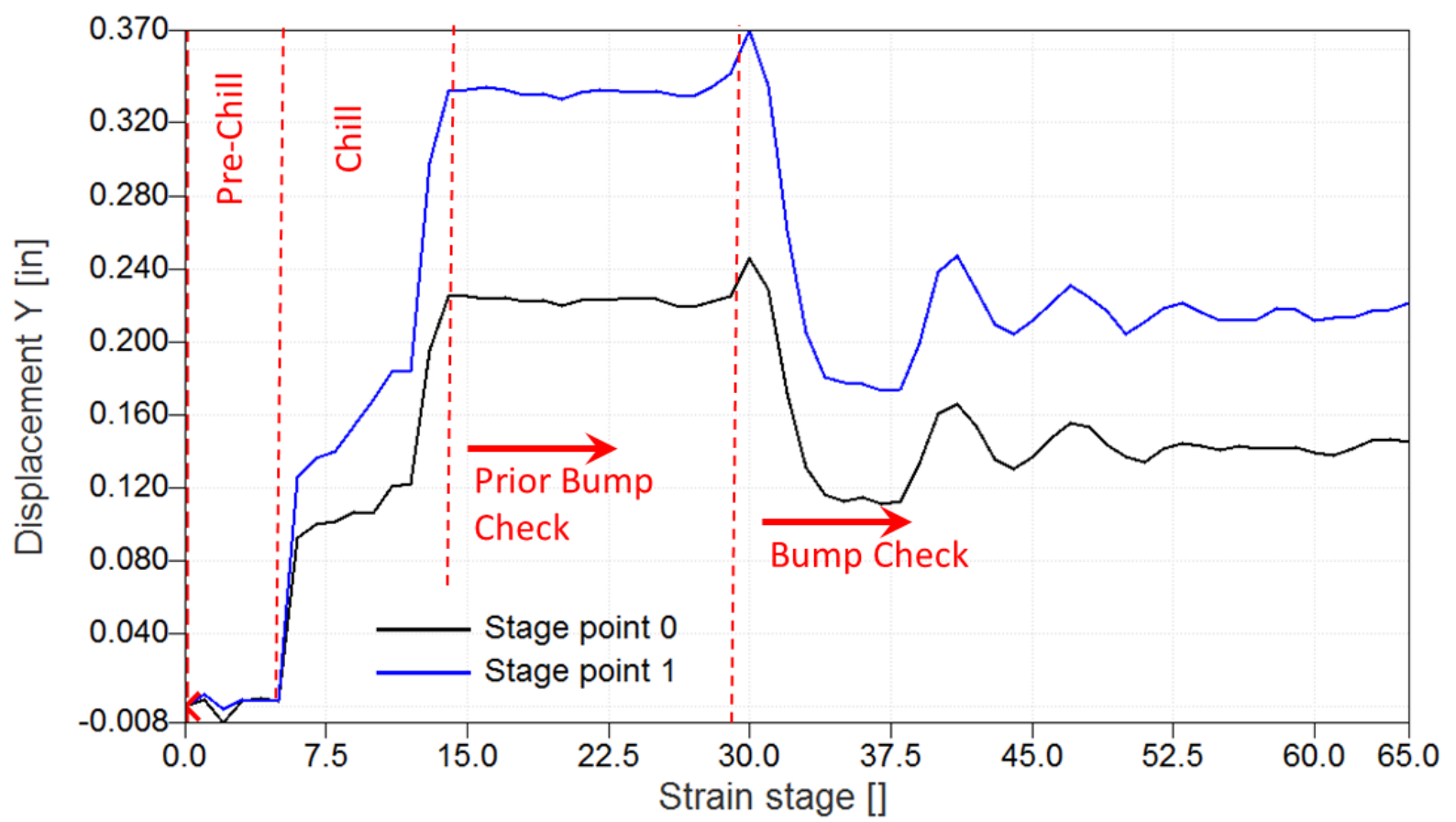

Figure 6. Pre-test Data collection with observed propellant loading, chill operations and gimbal checkouts.

The synced cameras were triggered during the hot-fire test sequence with a facility signal to ensure proper timing with the facility data system. The frame rate of this data collected with the Phantom v7 cameras was limited to the available onboard RAM. A framerate of $60 \mathrm{fps}$ was used to maximize duration of data collection and a corresponding image size of 512x384 pixels provided an adequate viewing area. Following pre-test and hot-fire test data collection, all images were exported as 8/24 TIFF images and imported into the ARAMIS software. It was observed that the exposure was lower than optimal for digital image correlation processing and, in some cases, postprocessing of images was used.

Lighting was fairly stable during pre-test data collection since the engine was protected, but varied slightly during engine start and increased slightly with initial exposure to the plume. Exposure and frame rates were established just prior to engine start since the cameras were tied into the facility trigger system for data correlation. Due to this added exposure during test potentially causing overexposure conditions, the software exposures were set darker than necessary. Post-test batch photo processing was used as a tool for underexposed (dark) images. A comparison was completed with various post-processing techniques and very small differences were observed in the absolute values of displacement and strain measurements. It should be noted that the pattern-matching algorithms in the ARAMIS software were fairly robust and could tolerate a higher degree of under-exposure than the user might expect. It was general practice to capture under-exposed images and post-process if needed. An example of the postprocessing technique is shown in Figure 7. 

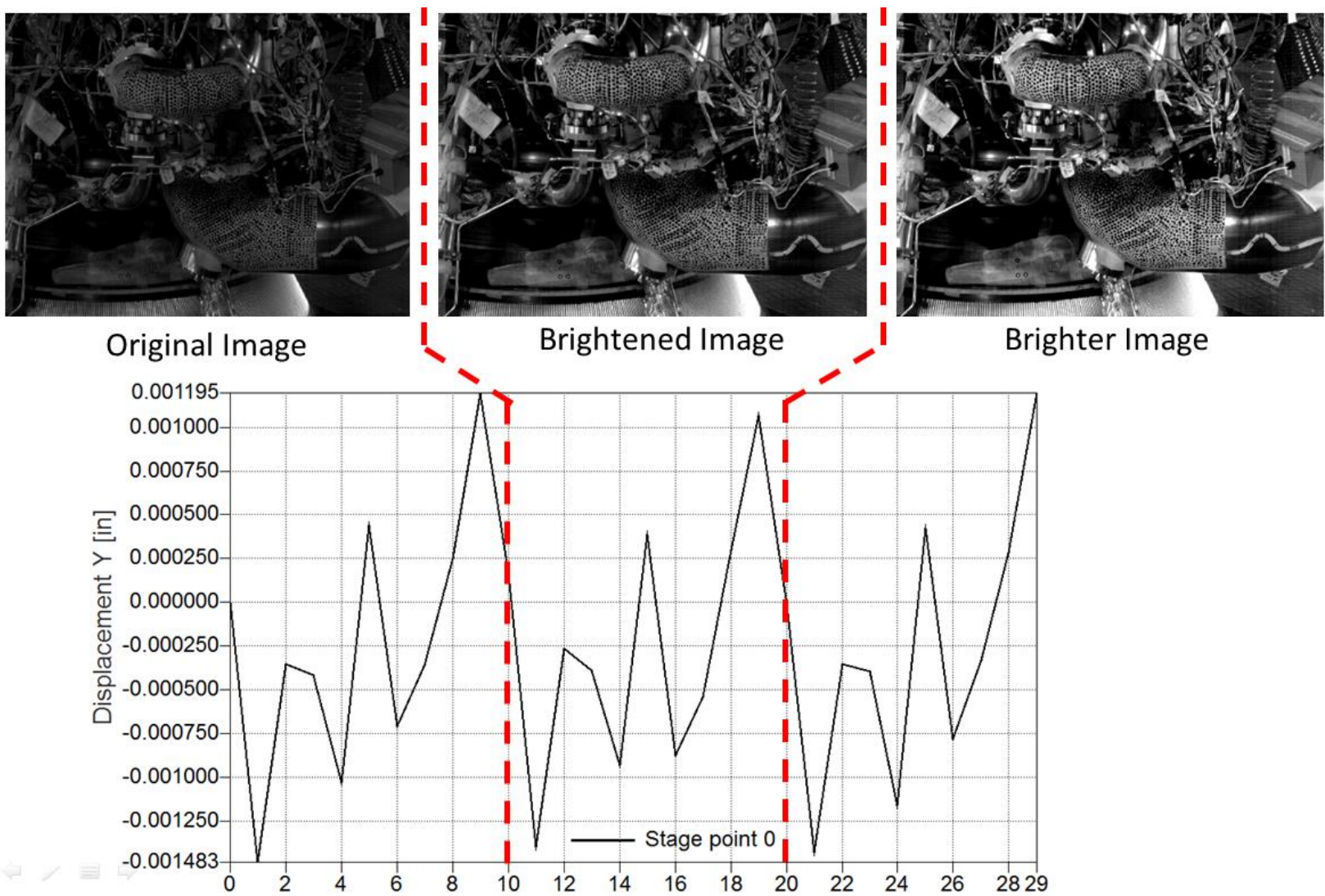

Figure 7. Comparison of post-processing image techniques; comparison of errors associated with image batch processing.

There were minor differences observed between the original reprocessed images. The differences observed in displacements were less than 0.0003 " and microstrains were less than $70 \mathrm{um} / \mathrm{m}$. These differences varied slightly for different sized facets and steps and also the resolution of images, but all with the same range. The images used for this study were fairly course at 512x384 pixels and would improve with higher resolution images and optimal facet sizing. Facet optimization was not a part of this study. The above differential values were beyond the system noise and error of the measurements, so it was concluded the post-processing did not have any effects on the data. The level of contrasting obtained from post-processing certainly aided in pattern recognition.

Initial processing of the test images revealed some challenges. The first observation was the extensive low density ice that liberated from the propellant lines above the engine during start. The ice debris and vapors partially blocked one or both camera views rendering images unusable at start at this location on the engine. These images were left as stages, but disabled for image processing. The ARAMIS software allowed for facet recognition to continue when both cameras had a clear view of the stochastic pattern after debris had cleared.

A facet size of $14 \times 14(\mathrm{X} \times \mathrm{Y})$ was used for processing and step size of $4 \times 4(\mathrm{X} \times \mathrm{Y})$ for all data processing to provide the $3 \mathrm{D}$ grid. Standard settings such as intersection deviation of $<0.5$ pixels was initially used, but later increased to 0.8 pixels due to some facet pairs that would not resolve in the highly dynamic environment. This increased the error in measurements, but was still below the system noise. Stages were processed manually since ARAMIS had occasional difficulties with automatic pattern matching. Complex start points were resolved in the software for each stage of pattern matching. An example of data collection from a time slice during test A2J028 is shown in Figure 8. 

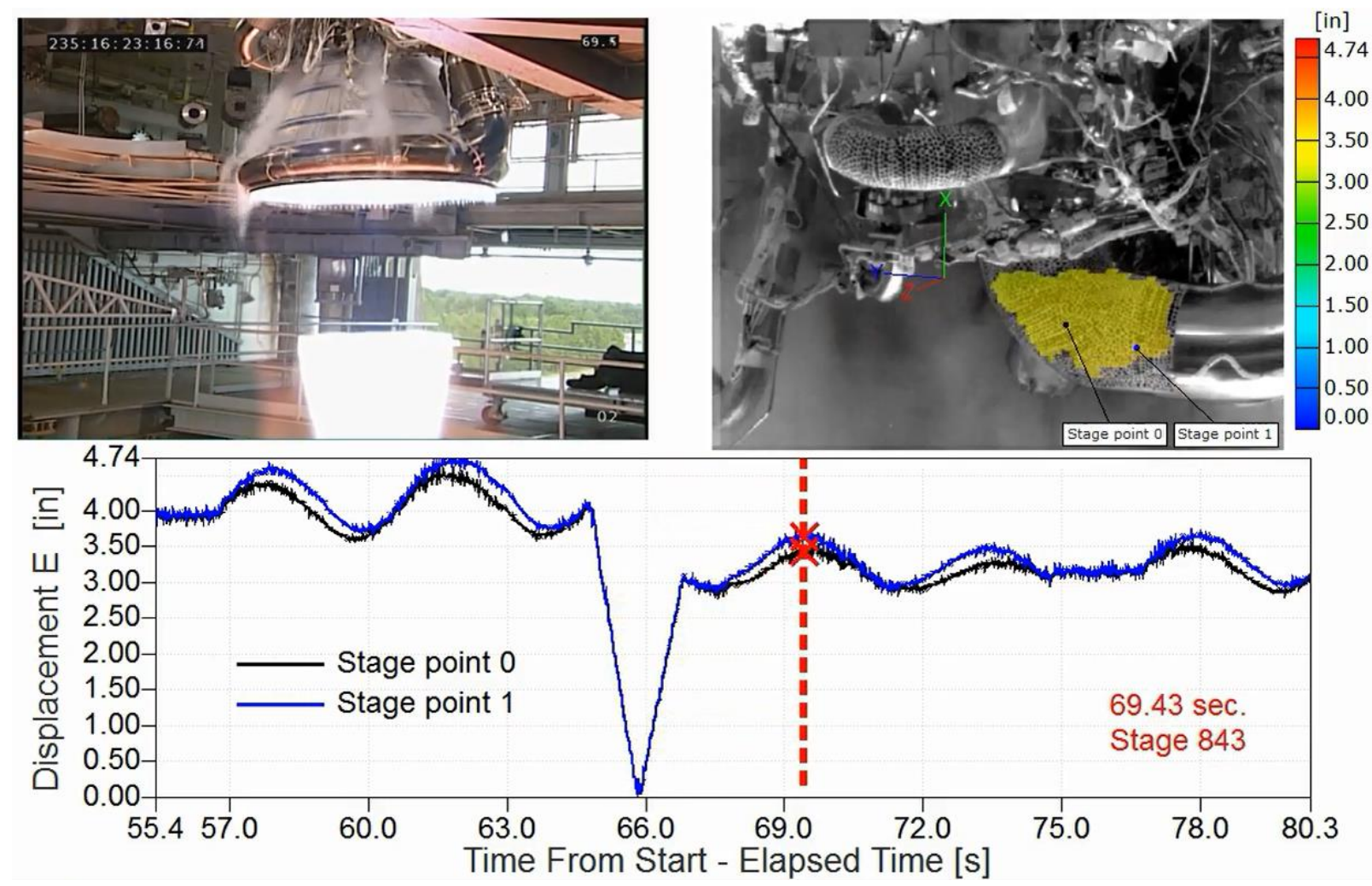

Figure 8. Snapshot of processed data from test A1J028 during gimbal operations showing total displacement of the engine.

Initial measurement error was determined during static test periods when the actuators (and subsequent gimbal movement) experienced a dwell. These were the periods within the dataset when total displacement should have been zero, but was not. There were some movements in total displacement shown in Figure 9. These movements were likely real since the engine experienced significant vibrations during hot-fire in addition to the cameras vibrating from facility interactions and loads. Since the cameras did not have any accelerometers attached during testing, it was not possible to distinguish the full source of error. Additional analysis of the data revealed that $15 \mathrm{~Hz}$ frequency content was dominant, in addition to the global gimbal movements.

Two different points were analyzed in further detail on the duct. The standard deviation of each static dataset was 0.017 and range of the data was identical at 0.0854 . Comparing the range of the local static dataset to the overall range of the global movement set was $1.9 \%$. A 6-period moving average was also applied to the static dataset. Some low frequencies may be present, but the data sampling rate was not high enough to make accurate conclusions. It is not known if the errors are real or attributed to engine or camera movement, but suspected a combination of both. 


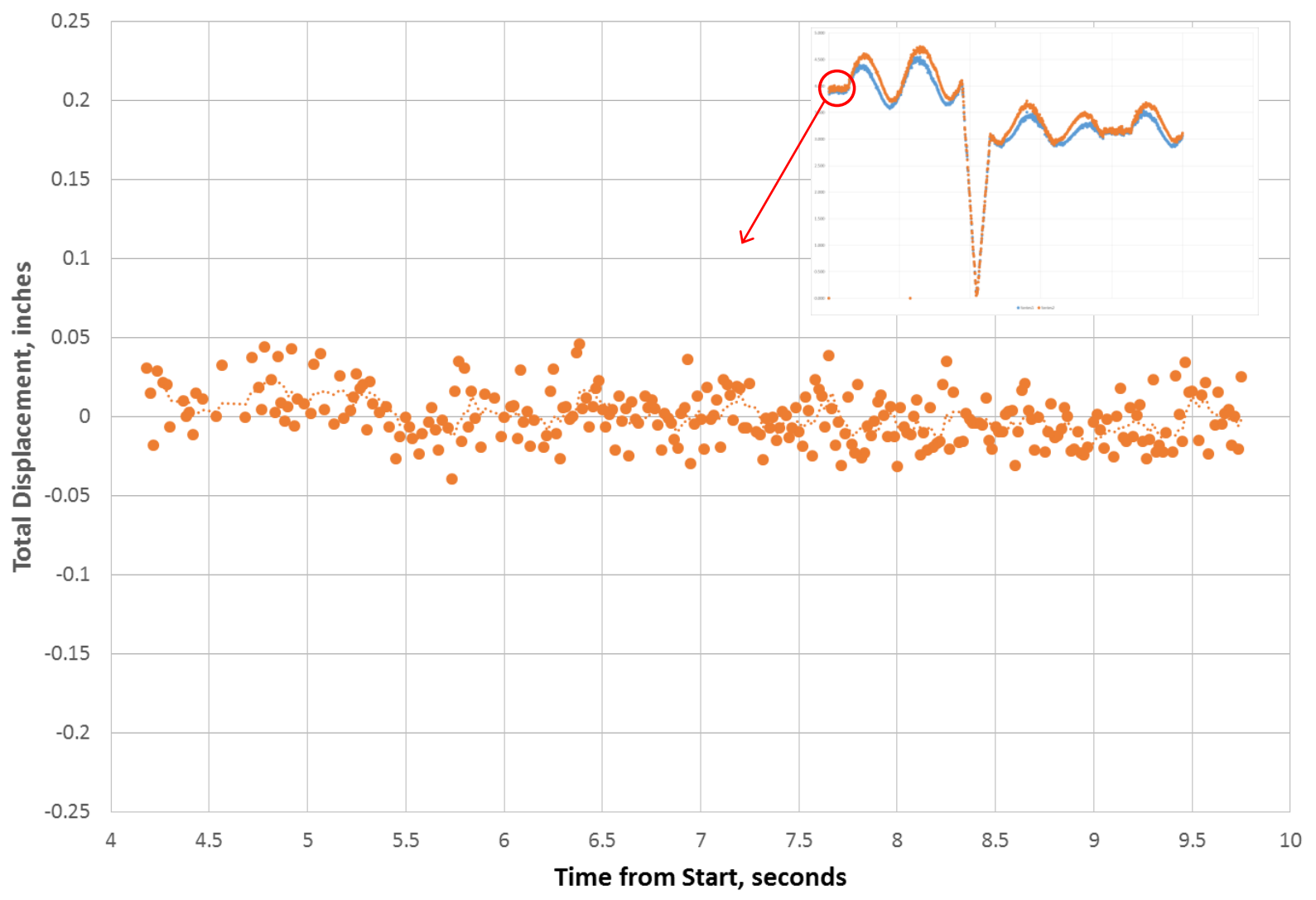

Figure 9. Vibrations and associated error observed during non-gimbal operation of the engine; $15 \mathrm{~Hz}$ frequency domain.

The data analyzed during hot-fire on the GGED and FTED included directional and total displacement, as well as directional, major, minor and Von Mises strain measurements. The majority of the data presented is unfiltered in ARAMIS, which increased some the noise level. However, the use of advanced filtering techniques was limited to the coarse grid structure on the components. It was mentioned earlier in the paper that filtering is a function within the ARAMIS software, as opposed to further post-test data reduction and offline filtering in another software package. This option is still available as the data can be exported in tabular format. An example of data collected during the test is shown in Figure 10. 

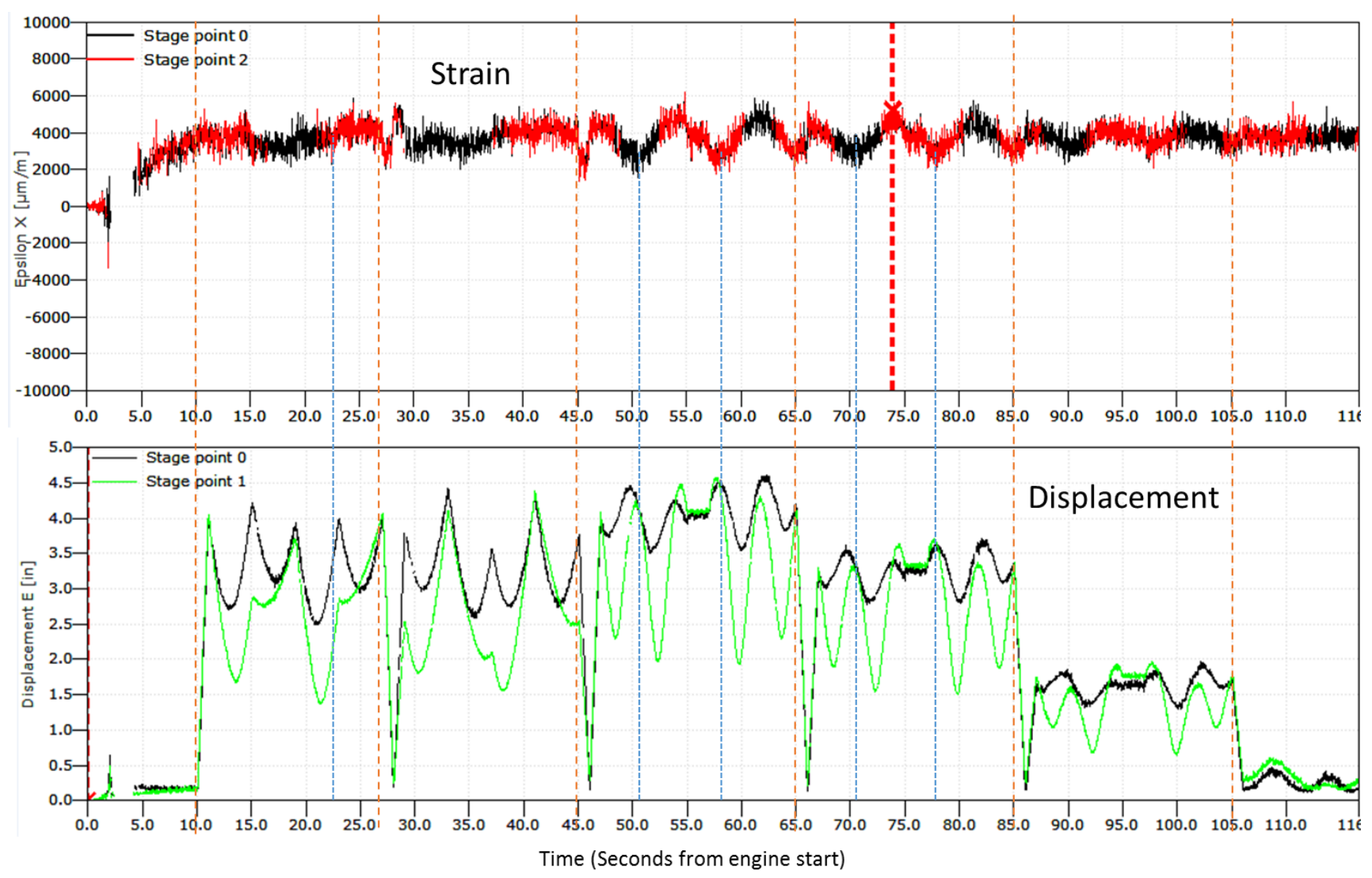

Figure 10. Raw data for major strain and total displacement of the gas generator (GG) duct during test A1J028.

The initial displacement data tracking the global gimbal motion matched predictions of the gimbal patterns and total displacement data shown in Figure 11. The directional strains and their alignments with the global gimbal movements are shown. The directional strains are observed when the forces are reacting through the turbopump housings and subsequent ducts. 


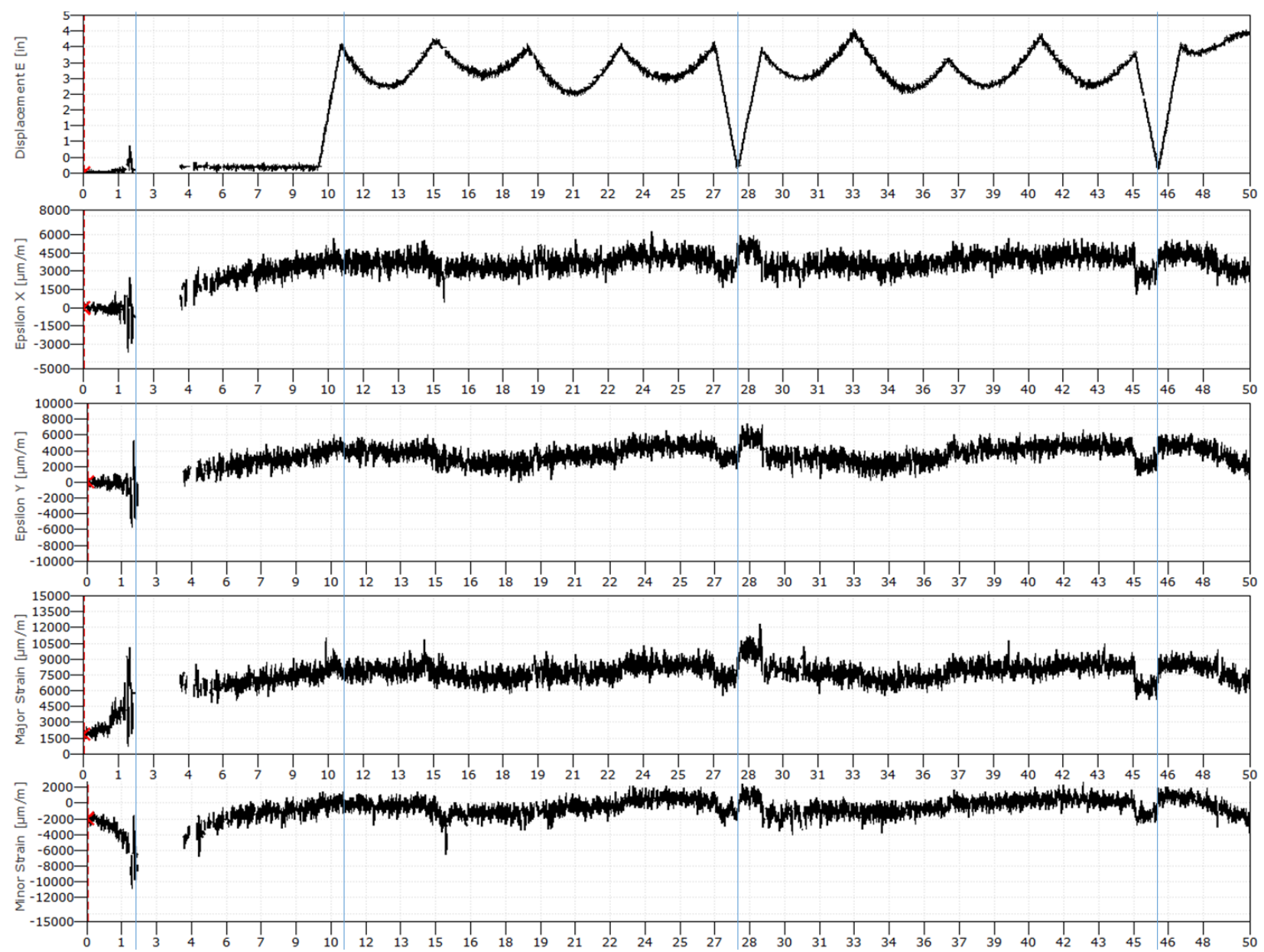

Figure 11. Comparison of direction, major and minor strains with total displacement on the Fuel Turbine Exhaust Duct.

The data observed in the plots show the local strains at the times of the global movements of the engine from gimbal maneuvers on the FTED. The dropout of data can be observed at time $=2-4$ seconds. This is due to ice debris from run lines and vapor events that cause one or both camera views to be blocked.

\section{Conclusion}

The digital image correlation technique provided a method to obtain non-contact optical measurements during hot-fire testing. The environment provided significant challenges for data collection due to the variety of low and high frequencies present on the engine and camera setup, in addition to elevated heat loads on the cameras and on the component surfaces being measured. An iterative data collection process was used to refine the technique for hardware setup, lenses, and camera operation that provided a stable and solvable pattern matching technique for the duration of the hot-fire testing. The setup provided some level of error in the measurement, but was at significantly lower levels than the measurements of interest.

Subscale and lab experiments proved valuable in both developing the process and in setting up offline, prior to expensive full-scale hot-fire testing. A series of stochastic patterning paints were also evaluated that can provide the random speckle pattern for a variety of environments and temperatures. Post-processing techniques were developed and evaluated to aid in underexposed images and allow for more robust solving of the entire test series. This allowed for additional data to be resolved during processing and limited errors in pattern recognition.

The digital image correlation hardware setup and techniques developed could be further refined to provide data collection on future engine testing and components. The primary purpose of advancing the technique was to measure 
liquid engine nozzle components and high temperature nozzle extensions. The testing presented in this paper provided early feasibility data to further develop the technique for nozzles and extensions and other high temperature components. Some limitations were discovered in the technique, including the ability to process large quantities of paired images. The software was limited to approximately 5,000 paired images (stages) per session and required additional processing resources. It is expected that future versions of the software will improve the allowable data and processor usage. This will be required for future testing since the high-speed camera frame rate of events would be expected to significantly increase.

Setup changes should be considered, such as instrumenting of the cameras in a highly dynamic environment so that the sources of error could be determined. Additional developments will also be evaluated to increase the temperature capabilities of the pattern matching to make use of infrared or ultraviolet techniques. Visible light filtering techniques may also be considered to make use of existing high-speed equipment.

\section{Acknowledgments}

The author would like to thank several contributors to the introduction and development of the D.I.C. technology for large scale rocket engine testing at NASA. Gilbert Handley under the Jacobs ESSSA contract was the key contributor and partner in this development program. Gilbert worked countless overtime and late night shifts aiding with design and iterations of the setup and process development. Mike Kynard and Mike Shadoan supported the development of this technology and funded advancement under the J-2X program and are now seeing how the investments have paid off for future programs. Thank you to the Stennis team, including Amanda Stein for integration into the test stand, Dan Goade for camera integration with existing systems, and the A1 technicians and machinists, who helped with installation, welding and enclosure fabrication. Thank you to the Rocketdyne team for helping with engine integration, including Mike Lauer's support of the technology and Brian Sproles. An additional thank you to Brian West for aiding with test A1J026 data collection.

\section{References}

${ }^{1}$ Tao X, Xu D, Zhang Z, Wang K, Qi X. Vibration Measurement in High Precision for Flexible Structure Based on Microscopic Vision. Robotics. 2016; 5(2):9.

${ }^{2}$ Image Correlation for Shape, Motion and Deformation Measurements: Basic Concepts, Theory and Applications. ISBN-10: 0387787461 Springer US; 2009 edition (April 22, 2009).

${ }^{3}$ Tyson, J., Schmidt, T., Coe, D., Galanulus, K., Validation and Iteration of Computer Models using Full-field Optical Methods. SAE 2006 World Congress and Exhibition: Experiments in Automotive Engineering (Part 4 of 6) Optical Techniques in Automotive Engineering II (April 4, 2006).

${ }^{4}$ ARAMIS (Version 6.3.1) [Computer Software]. Braunschweig, Germany: GOM mbH.

${ }^{5}$ PONTOS (Version 6.3.1) [Computer Software]. Braunschweig, Germany: GOM mbH.

${ }^{6}$ R.B. Berke, C.M. Sebastian, R. Chona, E.A. Patterson, and J. Lambros, "High Temperature Vibratory Response of Hastelloy-X: Stereo-D.I.C. Measurements and Image Decomposition Analysis. Experimental Mechanics. DOI 10.1007/s11340-015-0092-3. 2015.

${ }^{7}$ Grant BMB, Stone HJ, Withers PJ, et al. High-temperature strain field measurement using digital image correlation. Journal Strain Analysis Engineering 2009; 44(4): 263-271. 
${ }^{8} \mathrm{Pan} \mathrm{B}, \mathrm{Wu} \mathrm{D}$ and Gao J. High temperature strain measurement using active imaging digital image correlation and infrared radiation heating. Journal Strain Analysis Engineering 2014; 49(4): 224-232.

${ }^{9}$ R.B. Burke, J. Lambros Ultraviolet digital image correlation (UV-D.I.C.) for high temperature applications. Rev. Sci Instrum. 85(4), 045121. 2014.

${ }^{10}$ Pan, B., Wu, D., Wang, Z., \& Xia, Y. (2010). High-temperature digital image correlation method for full-field deformation measurement at 1200 C. Measurement science and technology, 22(1), 015701.

${ }^{11}$ Gradl, P. R. Application of Optical Measurement Techniques during Fabrication and Testing of Liquid Rocket Nozzles. Joint Army Navy NASA Air Force (JANNAF) Liquid Propulsion Systems (LPS) Conference. Nashville, TN. June 2015.

${ }^{12}$ Byrd, T. "The J-2X Upper Stage Engine: From Design to Hardware", 46th AIAA/ASME/SAE/ASEE Joint Propulsion Conference \& Exhibit, Joint Propulsion Conferences, AIAA 2010-6968. USA: AIAA, 2010.

${ }^{13}$ Vision Research PCC, Phantom Camera Control (Version 2.1) [Computer Software]. Wayne, New Jersey. 